Revue internationale P.M.E.

Économie et gestion de la petite et moyenne entreprise

\title{
Innovation et croissance économique: rôle et enjeux du financement des PME
}

\section{Loïc Belze et Olivier Gauthier}

Volume 13, numéro 1, 2000

URI : https://id.erudit.org/iderudit/1008670ar

DOI : https://doi.org/10.7202/1008670ar

Aller au sommaire du numéro

Éditeur(s)

Presses de l’Université du Québec

ISSN

0776-5436 (imprimé)

1918-9699 (numérique)

Découvrir la revue

Citer cette note

Belze, L. \& Gauthier, O. (2000). Innovation et croissance économique: rôle et enjeux du financement des PME. Revue internationale P.M.E., 13(1), 65-86. https://doi.org/10.7202/1008670ar
Résumé de l'article

À travers une double lecture, économique et financière, ce travail tente d'expliquer en quoi le lien établi, au plan théorique et empirique, entre innovation et croissance économique est sous-optimal en raison d'une inadéquation structurelle entre la demande des PME innovantes et l'offre du marché des capitaux. 


\title{
Notes de recherche
}

\section{Innovation et croissance économique: rôle et enjeux du financement des PME*}

\author{
Loïc BELZE \\ Olivier GAUTHIER \\ Université de Bourgogne
}

\author{
MOTS CLÉS
}

\section{Innovation - Croissance économique - PME Asymétrie informationnelle - Capital-risque - Financement}

\begin{abstract}
RÉSUMÉ
À travers une double lecture, économique et financière, ce travail tente d'expliquer en quoi le lien établi, au plan théorique et empirique, entre innovation et croissance économique est sous-optimal en raison d'une inadéquation structurelle entre la demande des PME innovantes et l'offre du marché des capitaux.
\end{abstract}

\section{LES AUTEURS}

Loïc Belze est titulaire d'un diplôme d'études approfondies (DEA) en gestion option finance et achève actuellement son doctorat au LATEC-CREGO. II est également chargé d'enseignement à l'Université de Bourgogne. Ses principaux domaines de recherche concernent les stratégies de financement et le marché des offres publiques d'achat et d'échange.

Olivier Gauthier est agrégé en économie et gestion, titulaire d'un DEA de sciences économiques et termine actuellement un doctorat au LATEC. II est également chargé d'enseignement à l'Université de Bourgogne. Ses recherches portent sur la théorie de la croissance économique et sur l'histoire de la pensée économique.

Adresse : LATEC, Pôle d'économie et de gestion, 2, boulevard Gabriel, 21000 Dijon.

* Les auteurs remercient Philippe Desbrières et Elie Sadigh, respectivement professeur et maître de conférences à l'Université de Bourgogne, pour leurs remarques faites sur une version préliminaire de ce travail présentée au IV Congrès international francophone sur la PME (CIFPME) à Nancy / Metz. Nous remercions également les évaluateurs anonymes ainsi que Pierre-André Julien pour leurs commentaires et suggestions. Les auteurs restent seuls responsables des erreurs ou omissions qui pourraient subsister. 


\section{ABSTRACT}

Through a both economical and financial approach, this working paper tries to go through the link between innovation and economic growth, in a theoretical and empirical way, stressing the sub-optimality rooted in the inadequation between demand of innovative SMEs and supply of the capital market.

\section{RESUMEN}

A través de una doble lectura, economica y financiera, este trabajo intenta explicar en qué este lace estalece, sobre el plan teorico e empirico, entre inovacion y recimiento economico, se revela suboptimal del hecho de una inadecuacion structural entre la demanda de las PyMEs inovantes y la oferta del mercado de los capitales.

\section{ZUSAMMENFASSUNG}

Aufgrund einer ökonomischen und finanziellen Sichtweise versucht diese Arbeit den Zusammenhang zwischen der Theorie und Empirie, zwischen Innovation und wirtschaftlichem Wachstum zu erklären. Die strukturelle Diskrepanz zwischen den Ansprüchen einer innovativen KMU und dem Marktangebot an Kapitalresourcen erweist sich als nicht optimal.

\section{Introduction}

La problématique du financement des investissements matériels et immatériels des PME à fort potentiel technologique constitue, depuis quelques années, un thème récurrent en sciences économiques et en sciences de gestion. L'objet de cet article est de mettre en exergue, dans le cas français, les conséquences d'une insuffisance structurelle dans l'octroi des financements nécessaires à la naissance et à la croissance des structures de production légères exerçant leurs activités sur ce type de segments. À travers une double lecture, économique et financière, nous montrons en quoi le lien établi, au plan théorique et empirique, entre innovation ${ }^{1}$ et croissance économique se révèle sous-optimal en raison d'un accès restreint des PME au marché des capitaux. Après avoir proposé une lecture macroéconomique de cette relation (section 1), nous tentons de rendre compte, à la lumière des théories financières récentes (section 2 ), des réelles perspectives et possibilités offertes à l'économie sous condition d'un rééquilibrage du système de financement de l'innovation, et ce, de la phase de préconceptualisation à la commercialisation (section 3).

1. Traditionnellement, l'innovation est définie comme l'application industrielle ou commerciale d'une nouveauté sur un produit, un procédé ou processus de production. Le processus d'innovation intègre toutes les phases depuis la R-D jusqu'à la production ou la commercialisation impliquant aussi bien les membres de l'entreprise que les partenaires extérieurs. Nous retenons cette dernière approche dans ce travail. 


\section{Innovation et croissance économique}

En matière de dynamique économique, l'allégeance des chercheurs au corps d'hypothèse walrasien avait occulté l'étude des conditions et effets de la génération du progrès technique dans un environnement capitaliste. Dans les modèles du type de Solow (1956), par exemple, la croissance économique se voit inhibée, en l'absence d'innovation, du fait de l'hypothèse de décroissance des rendements physiques marginaux du capital accumulé. Partant, l'évolution technologique ne peut être analytiquement intégrée que sous la forme d'une composante exogène, plus précisément sous la forme d'un stock. Depuis quelques années, la rupture paradigmatique d'avec le cadre walrasien standard a permis de considérer le changement technologique comme l'une des sources explicatives de la croissance des économies industrielles; ce type de modèle dit «à croissance endogène» tend à évoquer le progrès technique comme résultante d'une production de connaissances faisant l'objet d'une possible accumulation. Les travaux présentés dans cet article semblent en cela affirmer l'existence d'une relation positive entre degré d'innovation et croissance économique. Comme nous le mettrons également en évidence, l'approche microéconomique de ces théories semble constituer une base solide d'analyse du rôle de la PME innovante dans le procès de croissance.

\subsection{Apports théoriques récents}

Pour nombre de contributions récentes, la génération de l'innovation ainsi que sa diffusion sont déterminées par les comportements des agents et par des variables macroéconomiques (Guellec et Ralle, 1993). En l'occurrence, il s'agit de reconsidérer l'impact d'une action délibérée de recherche et développement (R-D) essentiellement financée par des fonds privés sur les fondements de nos économies. Cela suppose, en outre, d'étudier la génération de l'innovation comme conséquence d'une phase de R-D dont l'aboutissement demeure fonction du degré de productivité des chercheurs, mais aussi et surtout de l'existence et de l'importance du stock de connaissances globalement disponibles. L'approche que nous développons privilégiera les modèles mettant en exergue le rôle du «capital humain » dans la génération de l'innovation (Lucas, 1988), les modèles considérant l'innovation comme élargissement de la gamme de biens intermédiaires (Romer, 1990, 1991) et finaux ou, encore, de la qualité des produits existants (Grossman et Helpman, 1991 ; Aghion et Howitt, 1992).

\subsubsection{Capital humain, $R-D$ et croissance économique}

Les travaux de Lucas (1988) tendent à affirmer l'existence d'un corrélat fort entre niveau moyen de capital humain et productivité des facteurs de production. Comme le précise Muet (1994), dans le présent modèle, la productivité marginale du capital 
augmente avec le ratio du capital humain au capital physique, et, en présence d'externalités, elle augmente également avec le niveau de capital humain. Somme toute, il semblerait que les nations possédant un niveau élevé de capital humain puissent engendrer nombre d'effets externes positifs relativement à l'accumulation individuelle du capital humain. Amable et Guellec (1992) ont particulièrement insisté sur le fait que l'introduction de facteurs particuliers comme le «capital humain » ou la « connaissance » permet de contourner les problèmes qui se font jour au sein des analyses néoclassiques traditionnelles, car leur accumulation ne dépend plus du produit où se situent les rendements physiques marginaux décroissants. Le progrès technique est alors permis par l'accumulation des connaissances, variable dont le niveau par tête peut croître sans limite, à la différence du capital physique. Dans ce contexte, Romer (1990) souligne qu'une part majeure des connaissances accumulées ainsi que des externalités d'information liées à la diffusion de la connaissance provient de l'effort de R-D des firmes. En effet, un accroissement du capital humain employé dans ce domaine, comme un accroissement du niveau de connaissances global, tend à accroître la productivité des chercheurs. Partant, à long terme, la croissance économique semble pour partie dépendre de la capacité des activités de R-D (publiques et privées) à mobiliser leurs stocks respectifs de connaissances et de capital humain dans une perspective de génération rapide du progrès technique. À la suite de Arrow (1962) et Sheshinsky (1967), cette conclusion se présente comme d'autant plus valide si nous considérons que les processus d'apprentissage par la pratique décuplent également l'efficacité des structures susmentionnées. En matière de politique économique, toute politique de subvention à l'éducation et à la recherche, du fait qu'elle accroît le niveau moyen de capital humain et le stock de connaissances, est donc vecteur de croissance. À un niveau supérieur, ce phénomène d'expansion des connaissances prend un caractère intertemporel étant affirmée l'irréversibilité dont fait l'objet l'accumulation du savoir. À la lumière des diverses approches théoriques, l'existence d'un corrélat fort entre niveau moyen de capital humain, degré de connaissance et productivité de l'activité de R-D parait se dégager ${ }^{2}$. Cela n'est cependant pas suffisant pour expliquer, durant ce siècle, la croissance soutenue de la productivité totale des facteurs.

\subsubsection{Structure de propriété et incitation à la $R-D$}

Dans une économie capitaliste, l'innovation et la R-D privée effectuées de façon autonome sont motivées par l'espoir d'un rapide retour sur investissement sous condition d'existence d'une structure stable de propriété. De fait, l'incitation à la

2. Logossah (1994), dans une récension des évaluations empiriques du lien étudié dans ce travail, soutient que, faute de certitudes, de fortes présomptions existent du moins quant à la vraisemblance de l'effet positif de l'éducation et de la formation sur la productivité totale des facteurs. 
R-D privée, compte tenu du niveau élevé de risque inhérent au développement de nouveaux produits ou procédés, ne saurait s'orienter que vers les domaines assurant à la firme innovatrice un certain degré de monopole lui permettant l'exploitation de son droit de propriété industrielle ou servicielle. Afin de mieux cerner les éléments qui incitent les firmes à financer des programmes de R-D, Romer (1990) propose de classer les biens ou procédés produits par cette activité selon leur degré de rivalité et d'exclusivité. Ainsi, un bien ou procédé est dit «rival » $s$ 'il ne peut être utilisé que par une firme ou personne tandis qu'un bien ou procédé est qualifié « d'exclusif » si son propriétaire peut en interdire l'usage par un autre. Suivant cette typologie, un bien économique conventionnel serait à la fois rival et exclusif tandis que les biens publics seraient non rivaux et non exclusifs, c'est-à-dire non appropriables. En l'occurrence, et suivant cette distinction, l'activité privée de R-D devrait logiquement s'orienter vers le financement de produits ou procédés du premier type. Cependant, la mise en place d'un système de droit de propriété, même si elle peut constituer une incitation au financement de programmes de R-D nécessaires au développement et à l'exploitation de produits ou de procédés innovants, est loin d'assurer de façon permanente à la firme une position stratégique dominante sur son marché. Jacquier-Roux (1994) montre à ce propos qu'en matière de progrès scientifique l'information relative à l'innovation fait rarement l'objet d'une appropriation et tend à être considérée comme un bien public ou collectif. Deffains (1997) insiste, quant à lui, sur le fait qu'au plan théorique la mise en place d'un système de droits de propriété, en matière d'innovation, pour être favorable à l'ensemble de l'activité économique, doit poursuivre un double objectif : encourager les firmes à produire de nouvelles connaissances et faire en sorte que l'information relative à ces découvertes soit rendue publique. Il reste que, à la suite de Romer, le caractère au moins partiellement exclusif des biens ou procédés produits semble néanmoins à l'origine de la mise en chantier de nombreux programmes de R-D privés ${ }^{3}$.

\subsection{3. $R-D$, innovation et croissance: mécanismes de transmission}

Au plan macroéconomique, le problème qui se pose est de savoir dans quelle mesure, et par quel biais, l'innovation et le financement de programmes de R-D influencent l'évolution des niveaux de vie. Romer (1990) suppose qu'un effort délibéré de R-D de la part des firmes conditionne l'élargissement de la variété des biens de production intermédiaires existants et que cette variation est à l'origine de nouvelles productions sur le marché des biens finals. Dans ce cadre, la génération de nouveaux biens de production dépend, comme cela a déjà été abordé, du

3. Barro et Sala-I-Martin (1996) semblent également établir la pérennité du lien innovationcroissance dans les systèmes économiques protégeant la propriété intellectuelle. 
niveau de capital humain ${ }^{4}$ alloué aux activités de R-D des structures spécifiques privées. Comme la connaissance peut être accumulée sans limite, les rendements de l'activité de R-D peuvent être croissants ${ }^{5}$. Sur le plan macroéconomique, la croissance se manifeste par la diffusion de l'innovation dans l'économie, laquelle enclenche un processus dynamique d'accumulation des connaissances et d'expansion des champs technologiques. En effet, d'une part, l'apparition de nouvelles activités et méthodes de production étend et renouvelle sans cesse le tissu industriel, mais, de plus, chaque nouvelle découverte accroît elle-même le stock de connaissances disponible, accroissant ainsi le niveau moyen de capital humain et la productivité des chercheurs et agents. L'hypothèse restrictive posée par Romer est que l'apparition de nouveaux biens intermédiaires n'affecte pas la production des firmes exploitant ces derniers. Or, la plupart du temps, nous devons considérer que l'élargissement de la variété des produits existants provoque une externalité négative liée à la diffusion de l'innovation. Dans une perspective schumpétérienne, Aghion et Howitt (1992) proposent de considérer que l'amélioration de la qualité des produits existants érode, voire supprime, les rentes ou quasi-rentes de monopoles perçues par les firmes jusque-là leader sur leurs marchés respectifs. L'effet positif de la R-D sur le taux de croissance doit alors être nuancé, car l'effet en retour de l'innovation dépend de la façon dont la nouvelle technologie affecte, en termes d'emploi par exemple, la productivité et la rentabilité des activités existantes (voir Bouabdallah et Villeval, 1997).

\subsection{Innovation et croissance économique : quel dessein pour les PME?}

Entre l'approche de Romer et celles de Aghion et Howitt dont les contributions sont données pour significatives, une voie médiane semble pouvoir être trouvée étant évident que les effets précédents (accroissement de la variété de produits existants et accroissement de la qualité des produits) se produisent simultanément. À ce propos, Barro et Sala-I-Martin (1996) souligne qu'en matière de croissance économique le danger peut provenir, dans une économie décentralisée, du fait que l'effort de recherche fondamentale tendrait à devenir insuffisant alors que l'effort voué à la recherche d'améliorations conséquentes de la qualité des produits existants deviendrait trop important. Or, nombre de contributions tendent à montrer que l'innovation des entreprises de grande taille est essentiellement vouée à l'innovation « de métier » en raison du recentrage de leurs activités effectué ces dernières années. De fait, il s'agit pour les grandes structures de déléguer les programmes de R-D

4. Nous émettons certaines réserves quant à l'usage dans la littérature de ce terme.

5. Ce point central des contributions récentes en matière de croissance économique a fait l'objet d'une critique portant sur la cohérence interne de ces travaux. Voir à ce propos Sadigh (1995). 
qui ne relèvent pas de leurs compétences clés à de petites entités spécialisées, principalement des PME, dont les spécialités seraient trop coûteuses à maintenir de façon permanente au niveau de la firme (SESSI, 1998). Ce type de sociétés de recherche sous contrat ne représente cependant qu'une infime partie des activités de R-D. Malgré ce mouvement, les données du ministère de l'Économie, des Finances et de l'Industrie (SESSI, 1998) caractérisent une forte concentration de la R-D française puisque les PME/PMI ne participent qu'à hauteur de $13 \%$ aux dépenses de R-D avec $18 \%$ des effectifs dans ce domaine. Notons à ce propos que $83 \%$ des crédits publics, militaires, grands programmes technologiques et crédits incitatifs vont, en France, à 12 grands groupes et à leurs filiales. Le rôle de la PME technologique dans ce domaine reste donc, à ce jour, très modeste.

Pourtant, les travaux récents concernant les PME ont montré qu'elles ne sont pas découragées par les économies d'échelle réalisées par les firmes de grande taille (Audretsch, 1994). Jullien et Paranque (1995) ainsi que Joffre et Wickam (1997) ont d'ailleurs constaté des rentabilités similaires, voire plus élevées des PME par rapport à leurs homologues de plus de 500 salariés. Clay et Creigh-Tyte (1994) ont de plus établi la contribution majeure des PME en matière d'emploi ; conclusion corroborée, pour la France, par les statistiques du ministère de l'Économie, des Finances et de l'Industrie (SESSI, 1998). En matière de R-D, Acs, Audretsch et Feldman (1994) ont signalé que les structures de production légères exploitaient davantage les résultats de la recherche des firmes concurrentes que les grandes entités et profitaient mieux des découvertes universitaires en matière de recherche fondamentale. Il s'avère en ce sens, suivant en cela Lefebvre, Lefebvre et Bourgault (1996), que contrairement aux autres groupes, les PME dites «technologiques» mettent nettement plus d'accent sur les activités de recherche appliquées ainsi que sur l'amélioration des acquis scientifiques et technologiques. En outre, cette analyse démontre que ce type d'entreprise possède également une vision mondiale de son activité, ce qui en fait un formidable moteur potentiel de croissance. Les contributions qui précèdent démontrent ainsi amplement que les PME, si elles parviennent à financer leurs programmes de $\mathrm{R}-\mathrm{D}$, créent des synergies technologiques et financières égales sinon plus élevées à leurs concurrents de grande taille. Il demeure donc paradoxal, pour une nation comme la France, dotée d'un niveau moyen élevé de capital humain, de constater une insuffisance chronique du tissu des PME en matière d'innovation et de R-D. Sur le plan national, la faiblesse de la recherche est par ailleurs confirmée par les demandes de brevets qui sont essentiellement soumises par les Américains (29,9\%) suivis des nationaux (17,4\%). De plus, comme le souligne le rapport Guillaume (1998), la part mondiale des publications des laboratoires scientifiques français a progressé de $16 \%$ en 12 ans tandis que la part de la France dans le système du brevet européen a diminué de $17 \%$. En France, le niveau moyen élevé de capital humain ne génère donc que peu d'applications et procédés dans les domaines de l'industrie et des services. 
Il découle des arguments précédents que le potentiel des PME en matière d'innovation demeure largement inexploité ; le rapport Guillaume (1998) concernant l'innovation et la recherche technologique témoigne de ces insuffisances. En substance, le faible dynamisme des PME françaises sur le segment des hautes technologies proviendrait :

- de l'absence, voire la quasi-inexistence, de fonds d'amorçage ;

- de l'absence d'un environnement stimulant pour accompagner le chercheur ;

- du flou qui entoure sa situation juridique;

- de la faiblesse de la culture entrepreneuriale en France.

Ce dernier élément n'est pas acceptable. En effet, l'existence de forts profits potentiels dans les activités innovantes laisse augurer que, plus qu'un problème de culture entrepreneuriale, le manque d'entrepreneurs est avant tout lié à l'absence de financement de démarrage et à l'incapacité, pour de nombreuses firmes, d'accéder à des sources de financement autres que le contrat bancaire d'emprunt classique, alors que leur développement le nécessiterait. Alors que la PME technologique semble devoir, en matière de croissance, jouer un rôle majeur, il semble que, pour des raisons structurelles, le financement de la majorité des projets ne puisse être mené à bien. La section suivante entend fournir certaines explications à cet état de choses.

\section{Le financement de l'innovation dans les PME: le cadre des théories financières récentes}

\subsection{Spécificité de l'innovation dans la PME}

La genèse de l'innovation, qu'elle se situe au sein des grandes entreprises ou des PME, repose avant tout sur l'idée de confiance. La confiance est en effet à la base de toute relation contractuelle entre deux parties et, de ce fait, à l'origine de tout financement potentiel. Nous raisonnons ici sur l'idée d'innovation qui trouve son origine aussi bien dans la création d'entreprise que dans le projet innovant de rupture dans une PME déjà existante. La personne qui trouve l'idée innovante ou pilote le projet à la source de l'innovation est rarement celle qui peut fournir aussi la manne financière nécessaire au soutien et à la réalisation du projet, lui-même à l'origine du processus de croissance. Nous n'envisageons pas d'établir ici une typologie des PME françaises, qui au demeurant constituent un tissu relativement hétérogène, mais bien de faire ressortir les spécificités de l'innovation dans la PME et d'examiner, à terme, si elles sont une barrière au financement et, par conséquent, à la croissance. En effet, « les investissements qui ne peuvent faire l'objet d'une procédure de sécurisation en raison de leur spécificité et ceux qui ne peuvent engendrer 
une contrepartie matérielle rapide, tendent à trouver des conditions de financement plus difficiles. Au premier chef de ces investissements se trouvent les processus innovants » (Allegret et Baudry, 1996). Les auteurs de cette assertion donnent là l'essence des difficultés auxquelles les PME ont à faire face dans le cadre de leur processus d'innovation.

\subsubsection{Un déterminant fort, l'asymétrie informationnelle}

La PME se caractérise souvent par une structure de propriété familiale ou, tout au moins, par une structure de propriété restreinte et concentrée. Le corollaire de cette situation induit généralement pour la PME une existence hors marché financier sur lequel l'information est révélée par le prix des actions (en supposant hypothétiquement un marché efficient). Vos (1992), sur un échantillon de 209 petites entreprises non référencées sur un marché, illustre cette spécificité par le fait que les mesures traditionnelles du risque (B) pour les grandes entreprises ne valent plus pour les PME non cotées (Ang, 1991). Dans l'optique d'un financement externe, les petites entreprises sont donc soumises à une forte contrainte de production d'information pour obtenir la confiance des bailleurs de fonds d'emprunt et réduire ainsi l'opacité relative à leur activité prévisionnelle. Cette asymétrie informationnelle renforce la défiance des banques, par exemple, qui ne sont pas incitées à rechercher une information rare et coûteuse et qui conservent une position prudente en n'investissant pas dans la PME ou en octroyant des prêts à des taux beaucoup plus élevés que pour les grandes entreprises (effet de taille). Ainsi, selon Dietsch (1989), ce sont les entreprises les mieux disposées à soumettre des documents prévisionnels et à expliquer leur stratégie aux banques qui obtiennent les meilleures conditions de financement bancaire ${ }^{6}$. Certaines banques ont bien essayé de mettre en place des cellules internes d'information sur la rentabilité de la relation d'affaires avec les PME, mais les évaluations restent inadéquates. L'incidence sur les contrats de financement est directe, comme le montre une étude de St-Pierre et Allepot (1998) sur 97 PME dont les résultats « confirment que les grandes institutions sont maladroites avec les petites entreprises et qu'elles sont inaptes à évaluer correctement leur niveau de risque afin de rédiger des contrats de financement adéquats ». Une enquête de l'Association française des banques (AFB) [limitée aux prêts indexés sur le taux de base bancaire, qui concerne donc essentiellement les PME] a, par exemple, mis en évidence l'absence de liens étroits entre le coût du crédit et la cotation Banque de France (Picory, 1995).

6. Toutefois, l'hypothèse d'un rationnement supérieur du crédit pour les PME peut être nuancée dans la mesure où Paranque et Cieply (1997) montrent que cela n'a été constaté que pour les plus petites d'entre elles et, en particulier, sur l'endettement à long terme (sans distinction dans ce cas concernant les PME innovantes). 
Le problème d'asymétrie informationnelle entre les PME innovantes et les bailleurs de fonds potentiels réside non seulement dans la non-observabilité de critères objectifs nécessaires à l'évaluation du projet (historique de la firme trop court, produit en rupture radicale avec le savoir-faire de l'entreprise), mais également dans la difficulté de juger ce qui est observable. Nous pensons ici à la situation en fonds propres de l'entreprise : le faible apport en fonds propres peut, par exemple, représenter un signal négatif, car synonyme d'une moindre prise de responsabilité de la part de l'entrepreneur-dirigeant (Allegret et Baudry, 1996); les fonds propres sont en effet attachés à la notion de solvabilité : ils sont le «matelas de sécurité » qui permettra de «faire face » en cas de ralentissement conjoncturel de l'activité (rémunération différée). Paranque (1994) rappelle cependant que « chaque entreprise, en fonction de sa situation économique et commerciale, cherchera à optimiser le recours au financement externe selon le coût de celui-ci, mais aussi en fonction du degré d'autonomie qu'elle veut/peut sauvegarder et de ses projets de développement ». Il s'agira donc d'analyser la façon dont les motivations des acteurs peuvent influencer l'offre de capitaux aux PME innovantes.

\subsubsection{Risque et actifs spécifiques}

Le fait d'innover implique que l'on sorte du cadre même de la rente de situation et que l'on investisse dans un nouvel actif; ce nouvel actif s'entend au sens large, englobant à la fois le nouveau projet et toute la structure de production de ce nouveau projet (investissement dans de nouvelles compétences, de nouveaux outils de production, etc.). La notion de spécificité d'un actif est définie par son aptitude à être interchangeable ou transférable, par sa faculté de pouvoir être utilisé dans d'autres fonctions ou par d'autres utilisateurs (Williamson, 1988). En ne retenant d'abord que cette simple définition, il semble effectivement que la nature des éléments d'actif mis en jeu par l'entreprise puisse avoir un lien direct avec la notion de solvabilité future. L'optique du bailleur de fonds d'emprunt est de pouvoir diagnostiquer, en cas de liquidation, l'existence d'un marché permettant de vendre les éléments d'actif afin de récupérer ses fonds ${ }^{7}$. Dans ces conditions, la naissance de la PME innovante, contingente à l'obtention du prêt bancaire, semble compromise. Ces entreprises se retrouvent en effet devant un paradoxe. Williamsom (1988) montre, dans le cadre de sa théorie des coûts de transaction, que l'exigence du prêteur augmentera dans la même mesure que le degré de spécificité de l'actif, allant jusqu'au refus de financer. Le financement par capitaux propres, gelés dans l'entreprise, permet alors d'éliminer ce risque et de financer l'actif spécifique. La spécificité

7. Cette question devient moins pertinente lorsqu'il s'agit de l'innovation dans la grande entreprise qui possède déjà un actif conséquent et qui a su dégager des rentes sur les produits qu'elle fabrique. L'actif de cette grande entreprise peut donc avoir une valeur objective aux yeux d'acquéreurs potentiels. 
des éléments d'actif, et des garanties insuffisantes aux yeux des créanciers dans l'information transmise, influent donc négativement sur la probabilité de pouvoir générer l'innovation ${ }^{8}$. Il reste donc à comprendre dans quelle mesure l'offre de financement présente sur le marché français peut être en adéquation avec la demande de ces PME innovantes.

\subsection{L'offre de financement accessible aux PME innovantes}

Le soutien financier étant inhérent à la réalisation de l'innovation, nous examinons les différentes sources de financement afin d'en analyser ultérieurement la pertinence dans le cas des PME innovantes.

Nous avons pu constater que les fonds propres étaient une condition sine qua non au démarrage du projet d'innovation. Ces fonds peuvent se trouver par apport direct (fondateur, associés), par aides publiques, par diverses formes d'intermédiation (capital investissement) ou, encore, sur le marché boursier (nouveau marché) ${ }^{9}$. À notre sens, cette dernière hypothèse n'est pas pertinente ici, car cette phase du financement intervient pour consolider, voire faire aboutir le projet d'innovation. Quant aux aides publiques à l'innovation, elles reposent essentiellement sur les aides de l'ANVAR qui interviennent au début de la vie de la PME; elles sont très sélectives et dépassent rarement le million de francs. Les apports en fonds propres d'associés (business angels) sont également difficiles à obtenir, car ils reposent souvent sur des réseaux de relations informels difficiles à intégrer ; néanmoins, cette forme de financement s'organise et représente $9 \%$ des fonds levés en capital-investissement pour l'année 1996 en France ${ }^{10}$.

Avant d'en préciser la pertinence, il convient de comprendre les modes de financement correspondant aux phases de la vie de la PME innovante. Nous reprenons ici la typologie de Lachmann (1996) qui présente la vie de la PME innovante en cinq étapes. La phase de faisabilité ou de définition sera financée par une

8. Il est à noter que ce problème de financement au début de la vie de la PME peut également trouver ses racines ailleurs, comme le révèle Henri Guillaume, ancien président de l'ANVAR (Agence nationale pour la valorisation de la recherche), en disant : " $L$ 'épargnant français n'a pas la même culture et le système fiscal ne favorise pas ce type d'investissement. Jusqu'à un passé récent, les investisseurs français n'étaient guère convaincus que l'on pouvait gagner de l'argent en misant sur des entreprises innovantes. » Les Échos, mai 1998.

9. Ce descriptif rejoint parfaitement l'analyse de l'origine de la structure de financement des petites entreprises faite par Ang (1991, p. 9) : "Pour une nouvelle entreprise, les premières sources (de financement) sont: l'épargne du propriétaire et les emprunts personnels, les amis et relations, les banques locales et les sources liées aux petites entreprises (companies de capital-risques, SBIC, etc.). »

10. Source : European Venture Capital Association, 1997. 
combinaison d'apports personnels (ou autofinancement) et, éventuellement, de quelques appuis publics, comme la préexpertise et l'aide aux services de l'innovation de l'ANVAR. Dans la phase de création (démarrage), le financement est assuré essentiellement par le capital de départ, d'éventuels fonds publics (primes régionales à la création d'entreprises et d'emplois, etc.). Lors de la phase de la première croissance, le financement public reste important, mais il cède progressivement la place aux fonds privés spécialisés de haut de bilan (capital-risque) et, éventuellement, aux participations industrielles. Vient ensuite la phase de développement avec des projets ayant une plus grande visibilité et présentant des garanties de chiffres d'affaires, puis, enfin la sortie, nécessaire aux entreprises qui veulent poursuivre leur développement ou aller sur le marché international, ce qui peut nécessiter des dizaines de millions de francs de ressources extérieures (financements mezzanine).

Si cette approche permet de bien cerner les relations entre les étapes successives de la PME et les financements qui y sont associés, elle n'en demeure pas moins qu'une vision normative des pratiques observées. En effet, cette description illustre les comportements idéaux des entrepreneurs et des créanciers, ces derniers étant actifs, dans cette analyse, aux différentes phases de vie de l'entreprise. Cependant, nous sommes en droit de nous demander dans quelle mesure ces acteurs sont présents de façon uniforme sur tous les segments du développement de la PME innovante. L'étude réalisée par le cabinet Rise Conseil (1997) pour le compte du ministère de l'Industrie montre justement, en croisant l'importance du dossier (montant requis) et l'origine géographique des bailleurs de fonds, que l'adéquation n'est pas totale (figure 1). La forte spécialisation de ces fonds sur des étapes de vie très précises de la PME laisse certaines d'entre elles avec bien peu de ressources (déficit de financement).

Les banques restent les acteurs principaux du capital-investissement. Au cours des années 1980, elles ont été fortement impliquées dans cette activité et leur intervention a pris plusieurs formes par la création de filiales spécialisées par type de métier allant du capital-risque au capital-développement ${ }^{11}$. En 1995, les banques représentent $54 \%$ des capitaux engagés, mais le montant de leur contribution suit une pente déclinante. La diminution de ces participations bancaires rejoint naturellement l'idée de rationnement du crédit pour les PME. Cela amène alors deux remarques. Si les banques sont aptes à fournir des fonds, la forme contractuelle du crédit est-elle la forme optimale pour les entreprises innovantes dont il est ici question ${ }^{12}$ ?

11. Lyon Expansion PME pour le Crédit Lyonnais, ou Banexi Ventures pour la BNP par exemple.

12. Constant et al. (1991) indiquent effectivement que des contrats commerciaux de prêt financier relativement complexes et assurés sur les actifs des petites sociétés peuvent dans certains cas réduire, voire résoudre, les problèmes liés aux coûts d'agence de la dette. 


\section{FIGURE 1}

\section{Offre de fonds propres selon les besoins des entreprises}

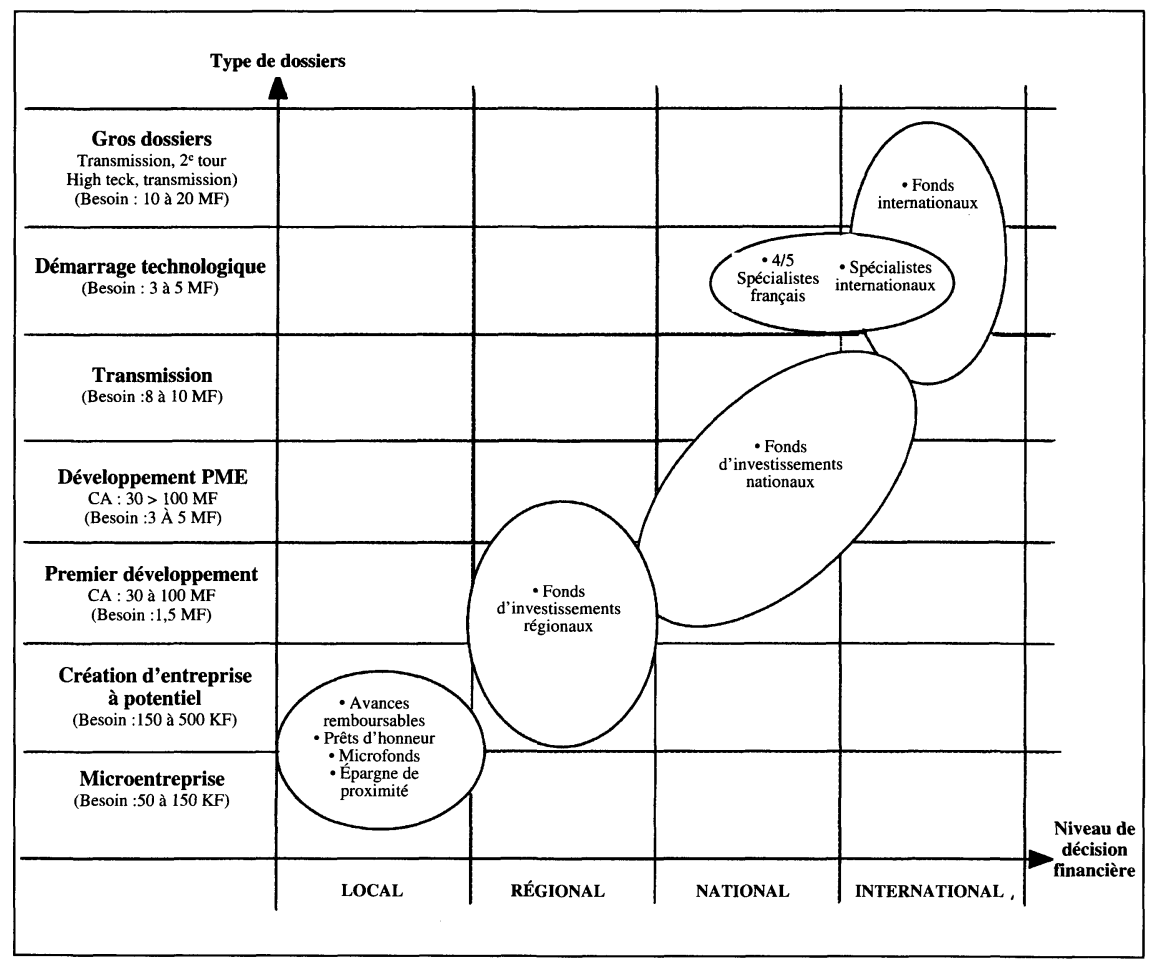

Source : Rapport pour le ministère de l'Industrie ; Rapport Rise Conseil, 1997.

Par ailleurs, Paranque et Cieply (1997) montrent que le rationnement du crédit ne touche que les plus petites PME et, spécialement, l'endettement à long terme. Les banques ont-elles alors les outils ${ }^{13}$ pour diagnostiquer la pérennité de ces PME innovantes afin de leur octroyer les fonds adéquats et nécessaires? Afin de mieux saisir les raisons du retrait des investisseurs institutionnels et l'inadéquation qu'il peut exister entre les demandes des PME innovantes et l'absence relative de bailleurs de fonds sur certains créneaux, nous présentons maintenant certaines grilles de lecture théorique qui nous permettront d'avoir une approche plus précise.

13. Nous pensons ici à des cellules internes de diagnostic spécialisées dans ce type d'entreprises. 


\subsection{Contraintes de financement de l'innovation chez les PME : quelle grille de lecture théorique?}

Une des conclusions de Colot et Michel (1996) à propos d'une théorie financière adaptée aux PME est que « la théorie traditionnelle, basée sur des hypothèses trop éloignées de la réalité des PME, ne peut servir utilement de référence. La pierre angulaire de la recherche demeure les progrès à réaliser dans les domaines de la science économique et de la théorie de la firme ». La grille de lecture demeure donc souvent mal adaptée. Une des pistes de recherche donnée par les auteurs précités nous aide à mieux comprendre " [les] relations entre demandeurs (entrepreneurs) et offreurs de capitaux (banques, investisseurs spécialisés dans le capital-risque)». Tout modèle ad hoc étant à exclure, si la PME posssède une structure de capital et organisationnelle différente de la grande entreprise, il demeure que le cadre théorique, empreint de la relation d'agence entre 1'entrepreneur et les créanciers s'appuyant ici spécifiquement sur la contrainte informationnelle forte ex ante et la contrainte de contrôle ex post, reste pertinent. Dans ce cadre, la constatation d'une domination encore grande du prêt bancaire dans le financement de l'innovation pourrait tenir à un intérêt propre à chacune des parties dans ce type de relation contractuelle. Du point de vue de l'entrepreneur, l'impact positif de l'obtention d'un crédit bancaire conséquent traduit les effets réputationnels dont il peut alors bénéficier (James, 1987), réduisant ainsi les problèmes de sélection adverse auprès d'autres créanciers (Diamond, 1991). Du point de vue du créancier, le contrat de dette «ne peut qu'inciter les dirigeants à être performants, vu le risque de faillite et de perte d'emploi qu'il engendre » (Desbrières, 1994). En conservant la pertinence de ce référentiel théorique, nous intégrons la nature spécifique de la PME innovante pour dépasser cette argumentation et proposer certaines réflexions sur l'état actuel de ce marché.

\subsubsection{Contrainte informationnelle}

Cette contrainte concerne la valeur perçue par les créanciers potentiels de l'information que la PME est capable de fournir. Il s'agit ici du problème connu sous le nom de «sélection adverse » : la demande de capitaux risque d'être désertée par les PME qui ont et pourront offrir la meilleure «qualité d'information ». Comme la banque ne connaît pas ex ante cette qualité, elle aura tendance à demander un prix élevé pour l'acquisition d'une créance ; il ne reste alors sur le marché que les PME qui ne peuvent trouver de fonds par d'autres voies.

La prise en compte simultanée des spécificités des PME et du caractère innovant de leur activité donne une importance extrême à l'asymétrie informationnelle (pas d'historique, pas de cotation) qui exerce une forte contrainte sur le financement. Dès lors, la théorie du financement hiérarchique de Myers et Majluf 
(1984) ainsi que les modèles théoriques sur la structure financière des entreprises $^{14}$ (basés sur le financement diversifié d'une grande entreprise) deviennent caducs. En effet, ces modèles s'appuient principalement sur des données mesurables (comptables, boursières) non disponibles pour les PME innovantes. Aussi, l'inadéquation entre l'offre et la demande sur ce marché semble donc être structurelle; or, la croissance et le développement de ce type d'entreprise sont contingents à cette adéquation.

Les banques restant les acteurs principaux de ce marché, le financement de l'innovation peut se faire a priori schématiquement de deux façons, par emprunt bancaire ou par apport en fonds propres. Dans le premier cas, cela implique pour l'entreprise des paiements fixes quel que soit l'état d'avancement du projet, alors que dans le deuxième cas, l'investissement en fonds propres (type capital-risque) implique généralement que le bailleur de fonds retire son investissement, assorti de la plus-value à l'issue du programme d'investissement. L'importance de la priorité de la créance prise par le bailleur de fonds dans le cas d'une faillite de la firme implique alors, comme le montre Winton (1995), une différence sur le degré d'asymétrie informationnelle entre les cocontractants lors de l'investissement initial. Nous comprenons le souci plus marqué des créanciers résiduels d'obtenir le plus d'informations possible pour contrôler le bon déroulement du programme d'investissement de l'entreprise.

À partir de cette alternative, nous pouvons altérer la santé du contrat d'emprunt bancaire pour les PME innovantes. En effet, l'offre de ce type de contrat découle essentiellement d'une logique de rentabilité (à court terme) pour la banque. Par ailleurs, l'implication bancaire sera toujours contingente au rang de priorité en cas de liquidation. Il est nécessaire pour la banque de pouvoir optimiser les coûts d'influence dans ce cas (Welch, 1997) ou, encore, de pouvoir permettre des renégociations plus efficaces s'il existe plusieurs bailleurs de fonds (Bolton et Scharfstein, 1996). La prise de participation en fonds propres demeure par ailleurs toujours assortie de conditions restrictives concernant les autres créanciers de l'entreprise, comme le montre James (1995) dans le cadre d'une renégociation de dette ${ }^{15}$. L'argument de spécificité des actifs développé par Williamson (1988) prend ici également tout son sens. Ainsi, Choate (1997) fait clairement ressortir, à partir d'une revue d'études empiriques, la corrélation qu'il peut exister entre le financement par endettement et le caractère redéployable des actifs financés. Cette anticipation ex ante du mode de financement par la banque reste, comme nous allons le voir, jointe à la notion de contrôle.

14. Voir pour cela la synthèse faite par Harris et Raviv (1991).

15. La banque craint de voir sa créance « juniorisée », ce qui implique un transfert de richesse potentiel vers les créanciers de moindre priorité. 


\subsubsection{Contrainte de contrôle}

L'analyse se fait ici ex post, c'est-à-dire la banque ne sait pas si la PME agira dans l'intérêt de son créancier, autrement dit si le crédit octroyé sera utilisé de façon optimale; c'est le concept de «risque moral». Dans le cas de la PME innovante, la banque étant incapable d'analyser le risque de défaillance, la seule méthode pour faire face est de garder un rang de priorité élevé et d'assortir le prêt d'un taux d'intérêt supérieur à celui qui aurait été octroyé aux grande entreprises. On doit retenir également que le contrôle entraîne des coûts pour le bailleur de fonds d'emprunt, qui doit alors faire un arbitrage ex ante entre le coût de contrôle et la rentabilité espérée du projet d'innovation ex post.

Si nous adoptons maintenant la perspective de la PME, le financement par emprunt bancaire présente des coûts et des bénéfices. Le bénéfice tient essentiellement dans l'espoir d'une renégociation plus aisée dans le futur. Cela passe par exemple par le développement de relations de clientèle, c'est-à-dire une relation de crédit fondée sur la répétition ; cette relation tient alors compte des spécificités de chaque entrepreneur (Psillaki, 1995). Au regard du coût, il s'agit essentiellement du problème de monopole de l'information que peut détenir une banque sur une PME en étant son seul créancier (problème de hold up) ; cela donne effectivement un pouvoir de négociation accru à la banque sur les bénéfices de l'entreprise une fois le projet d'innovation mis en place, comme le montre Rajan $(1992)^{16}$. De plus, les PME subissent le biais existant dans le financement à court et moyen terme, les banques n'étant pas pourvues de services de contrôle spécialisés dans ce type d'entreprises (Von Thadden, 1995). Ces coûts de contrôle peuvent tout simplement décourager les banques classiques (de dépôt), d'où la création par ces grandes banques de filiales spécialisées (Banexi, par exemple) de façon à profiter d'économies d'échelle concernant la spécialisation et l'expérience accumulée des experts. Le compromis pourrait alors se trouver dans l'établissement de modes de financement incitatifs pour les deux parties : une prise de participation dans les fonds propres de la PME innovante en sus d'un prêt à haut rendement pour la banque (Stiglitz, 1985) et des contrats incitatifs bien définis pour l'entrepreneur tenant compte de la qualité du projet et de son aversion au risque (Ravid et Spiegel, 1997) ${ }^{17}, 18$. La mixité des intérêts dans une participation donnée pourrait effectivement accroître

16. Voir également à ce sujet Houston et James (1996).

17. Les auteurs montrent également que seuls les entrepreneurs participant au capital du projet ont la possibilité d'obtenir un financement.

18. Il faut garder à l'esprit que ces entrepreneurs acceptent souvent, lors du lancement, des salaires moindres comparativement à ce qu'ils pourraient trouver dans une autre structure déjà existante. 
le degré d'implication. À la lumière des grilles théoriques mises en exergue, nous caractérisons maintenant le marché français du capital-investissement et développons une approche critique fondée sur le lien innovation-croissance.

\section{Activation du levier innovation-croissance: réflexions sur les stratégies de financement des PME françaises}

Le manque de capitaux « patients » est la faiblesse chronique du système de financement français. Nous pensons particulièrement ici aux fonds de pension qui sont quasiment inexistants en France. Or, les « démarrages ${ }^{19}$ » technologiques, moteurs de l'innovation et de la croissance, ont des besoins en capitaux qui augmentent très rapidement et cette obligation de croissance parfois très rapide réclame des professionnels très pointus et différents à chaque étape. Cette chaîne de financement est bien huilée aux États-Unis, où existe un réseau d'acteurs qui couvre tous les montants et tous les niveaux de risque, depuis le capital d'amorçage qui signe la naissance de l'entreprise jusqu' au marché financier qui assure sa consécration. Cette constatation résume assez bien la spécificité de l'identité de la PME innovante ainsi que, par contraste, les lacunes présentes dans le système de financement français.

Les pouvoirs publics avaient donné l'impulsion nécessaire par la création des SDR (Société de développement régional), des SFI (Société financière d'innovation), des IRP (Instituts régionaux de participation) et, finalement, des SCR (Société de capital-risque) et des FCPR (Fonds communs de placement à risque), mais nous assistons depuis 1992-1993 à une restructuration et à un repositionnement du métier qui se traduit par la disparition de certains fonds. Cette situation aurait essentiellement pour source la mauvaise conjoncture économique qui a augmenté le taux de sinistre, la difficulté pour les fonds d'assurer la liquidité de leurs participations et de trouver des « sorties » rentables ainsi que l'absence d'une épargne à long terme (Rapport Rise Conseil, 1997).

Les acteurs de ce marché sont essentiellement représentés par trois catégories d'investisseurs en raison du rôle prépondérant qu'ils jouent dans l'alimentation du système. Nous avons vu que les banques représentaient encore la moitié des investissements, mais que leur participation diminuait. Néanmoins, elles restent présentes avec la création de filiales spécialisées dans le métier de capital-risque (Crédit Lyonnais, BNP, etc.). Nous assistons toutefois à un recentrage de leurs interventions en raison de la difficulté d'exercer le métier de banquier commercial et celui d'investisseur en fonds propres. Il semble ainsi que les filiales en fonds propres des grandes banques à réseaux, en période de mauvaise conjoncture, puissent entrer dans

19. Traduction du mot «start-up». 
une phase de gestion particulièrement active de leurs participations et de sélection accrue dans leurs nouveaux investissements. Les assurances et caisses de retraite jouent un rôle mineur. En l'absence d'un système de retraite par capitalisation, la contribution des caisses de retraite ne peut être que marginale. Plus surprenante est la faiblesse des investissements des compagnies d'assurances si nous les comparons aux montants collectés par l'assurance vie ${ }^{20}$. Enfin, les industriels semblent s'être retirés du marché du capital-investissement. Leur participation se justifiait notamment dans le cadre d'une veille stratégique en participant à des fonds de capital-risque technologique, ainsi que par la volonté d'une diversification de leurs placements de trésorerie. Cependant, les résultats escomptés ne sont pas présents, car le marché du capital-investissement nécessite une forte concentration des investissements de même qu'une très forte expertise technique que n'ont pas fournies les grands industriels. Il convient enfin de remarquer que bien que la France soit le deuxième acteur sur le marché européen, le montant de ses investissements sur ce marché reste en 1996 plus de trois fois inférieur à celui de la Grande-Bretagne.

De là, nous touchons la pierre angulaire du système de financement de l'innovation par le capital-risque en France, source de la croissance du tissu des PME: les acteurs, leurs motivations et leurs incitations. Les banques, qui restent les acteurs principaux, recherchent aujourd'hui une rentabilité de leurs investissements à court terme en se recentrant sur leurs métiers d'origine; elles sont contraintes par la réglementation prudentielle et doivent dégager des bénéfices. De plus, la privatisation du secteur bancaire renforce cette tendance; les actionnaires privés n'apporteront des fonds qu'à la condition que ces dernières soient en mesure de satisfaire leurs exigences de rentabilité (Bancel, 1996). Il y a eu ainsi un véritable effondrement du capital-création en France qui concerne les capitaux d'amorçage et de démarrage censés assurer une part importante des financements en amont des projets d'innovation. Selon une étude de l'Association française des investisseurs en capital (AFIC) de 1994, citée par Lachmann (1996), les participations en faveur des nouvelles entreprises sont passées de 456 millions de francs en 1991 à 273 millions en 1994. Ce désengagement bancaire ne laisse alors souvent comme alternative que les prêts d'honneur ou les financements publics plus restreints, comme les aides à la création d'entreprises de l'ANVAR.

Nous pouvons par ailleurs remarquer qu'en dépit du mouvement d'innovation financière qui a conduit à la multiplication des instruments de financement proposés aux entreprises dans le cadre d'un marché de capitaux globalisé, une grande partie des PME est restée en marge de cette évolution, avec toujours une large domination du prêt bancaire parmi les ressources dont elles disposent (Levratto, 1994). Au

20. Cela est principalement dû au fait que les assurances ont développé leurs propres outils d'investissement en fonds propres et que leurs produits d'épargne à taux garantis les contraignent à investir dans des produits de taux plutôt que des produits d'actions. 
regard de cet élément, les PME innovantes doivent pallier les problèmes d'asymétrie informationnelle et de contrôle. L'amélioration du système de financement de ces sociétés non cotées que sont les PME passe par une modification de la relation bancaire; la banque doit dépasser le stade de la simple relation de clientèle pour atteindre une véritable relation de confiance. Cela pourrait se traduire par une concentration accrue des participations en fonds propres au niveau d'intermédiation supplémentaire que représentent les fonds spécialisés en capital-risque. Ces derniers permettent effectivement une économie sur la diversification de ce type de risque $^{21}$ et sur l'expérience accumulée des spécialistes qui les composent. Enfin, l'idée de «continuité territoriale du point de vue des solidarités de financement » mise en exergue par Allegret (1995), nous semble également importante dans le rétablissement d'une adéquation de l'offre et de la demande concernant les PME innovantes. Une certaine homogénéisation de la présence des investisseurs publics et privés, locaux et nationaux sur le territoire serait donc à même de renforcer les coalitions et d'accroître la flexibilité des stratégies de financement de l'innovation ${ }^{22}$.

\section{Conclusion}

À l'aune des développements précédents, la capacité des économies à activer le lien établi entre croissance économique et innovation demeure donc largement dépendante de la mise en ouvre d'actions significatives en ce qui concerne le financement des PME actives sur des segments de marché à fort potentiel. En l'occurrence, il s'agit de construire un système de financement des PME technologiques mettant fin au rationnement dont elles font l'objet sur le marché des capitaux. Cela passe, nous l'avons montré, par un redéploiement à l'échelle nationale et une homogénéisation des structures vouées à ce type de financements, permettant d'accompagner les structures de production légères à tous les stades de leur évolution et, plus particulièrement, au moment de leur naissance; car s'il semble juste qu'au plan macroéconomique, comme le précisent encore Fortin et Helpman (1995), les politiques économiques favorisant un niveau élevé de capital humain, soutenant la R-D et assurant l'accès aux connaissances et aux marchés internationaux contribuent pour une part non négligeable à la croissance économique, une approche plus fondamentale nous rappelle cependant que la genèse de la dynamique économique se révèle avant tout fonction de la capacité des nations, et donc des entreprises, à financer l'accumulation du capital. Or, à l'instar des PME innovantes, le cas français est à ce jour emblématique des insuffisances en ce domaine.

21. Ce risque peut être défini par des projets à forte valeur ajoutée concernant des PME contrôlées souvent par une personne ou une famille, avec peu de garanties sur actifs (collateralization).

22. Nous pensons notamment au rétablissement d'antennes locales pour les fonds d'investissement nationaux. 


\section{Bibliographie}

ACS, Z.J., D.B. AUDRETSCH et M.P. FELDMAN (1994), «R-D spillovers and recipient firm size », Review of Economics and Statistics, vol. 76, n ${ }^{\circ} 2$, mai, p. 336-350.

Aghion, P. et P. HowitT (1992), «A model of growth through creative destruction », Econometrica, vol. 60, $\mathrm{n}^{\circ}$ 2, p. 323-351.

AllegRET, J.P. (1995), «Le financement des PME innovantes : sous quelles conditions les technopoles peuvent-elles constituer une réponse adéquate ? », Revue internationale PME, vol. 8, $\mathrm{n}^{\text {os }} 3-4$, p. 149-163.

ALLEGRET, J. et B. BAUDRY (1996), «La relation banque-entreprise : structures de gouvernement et formes de coordination », Revue française d'économie, vol. 11, $\mathrm{n}^{\circ} 4, \mathrm{p}$. 3-36.

AMABLE, B. et D. GuELleC (1992), «Les théories de la croissance endogène », Revue d'économie politique, vol. 102, no 3 , p. 314-277.

ANG, J. (1991), «Small business uniqueness and the theory of financial management », The Journal of Small Business Finance, vol. 1, $\mathrm{n}^{\circ}$ 1, p. 1-13.

ARROW, K.J. (1962), «The economic implications of learning by doing », Review of Economic Study, vol. 29, p. 155-173.

AUDRETSCH, D. (1994), «Small business in industrial economics : the new learning », Revue d'économie industrielle, vol. 67, p. 21-39.

BANCEL, F. (1996), «Les stratégies de banque-industrie », Revue d'économie industrielle, vol. 77 , p. $29-52$.

BARro, R. et X. SAla-I-MARTIN (1996), La croissance économique, Paris, Ediscience international.

Bolton, P. et D. SCharfstein (1996), «Optimal debt structure and the number of creditors », Journal of Political Economy, vol. 104, n 1, p. 1-25.

Bouabdallah, K. et M. VilleVal (1997), «Innovation et croissance des inégalités sur le marché du travail », Revue d'économie politique, vol. 107, $\mathrm{n}^{\circ}$ 5, p. 567-605.

CHOATE, G. (1997), « The governance problem, asset specificity and corporate financing decisions », Journal of Economic Behavior \& Organization, vol. 33, p. 75-90.

ClAY, N. et S.W. CREIGH-TYTE (1994), «SMEs and employment in the European community : an industrial perspective ", Revue d'économie industrielle, vol. 67, p. 71-88.

COLOT, V. et P.A. MiCHEL (1996), «Vers une théorie financière adaptée aux PME. Réflexion sur une science en genèse », Revue internationale $P M E$, vol. $9, \mathrm{n}^{\circ} 1, \mathrm{p} .143-166$.

CONSTANT, R., J. OSTERYOUNG et D. NAST (1991), «Revolving asset-based lending contracts and the resolution of debt-related agency problems », The Journal of Small Business Finance, vol. 1, n ${ }^{\circ}$ 1, p. 15-28.

DEFFAINS, B. (1997), « Progrès scientifique et analyse économique des droits de propriété intellectuelle », Revue d'économie industrielle, vol. 79, p. 95-118.

DESBRIÈRES, P. (1994), « Nouvelles formes de fonds propres et gouvernement de l'entreprise ", Revue d'économie financière, hiver, p. 81-108. 
DIAMOND, D.W. (1991), « Monitoring and reputation : the choice between bank loans and directly placed debt », Journal of Political Economy, vol. 99, n 4, p. 689-721.

DIETSCH, M. (1989), «Les PME et les conditions bancaires », Revue d'économie financière, $\mathrm{n}^{\mathrm{0}} 10$, juillet-septembre, p. 72-85.

FORTIN, P. et E. HELPMAN (1995), «Innovation endogène et croissance : conséquences du point de vue canadien », Industrie Canada, Hors série $n^{\circ} 10$.

GUELLEC, D. et P. RALLE (1993), «Innovation, propriété intellectuelle, croissance », Revue économique, vol. 47, p. 319-334.

Grossman, G. et E. Helpman (1991), Innovation and Growth in the Global Economy, Cambridge, Mass., MIT Press.

HARRIS, M. et A. RAVIV (1991), «The theory of capital structure », The Journal of Finance, vol. 46, p. 297-355.

Houston, J. et C. JAMES (1996), «Bank information monopolies and the mix of private and public debt claims », The Journal of Finance, vol. 51, $\mathrm{n}^{\circ}$ 5, p. 1863-1889.

JACQUIER-ROUX, V. (1994), «De l'indivisibilité à la divisibilité de la R-D industrielle», Revue d'économie industrielle, vol. 68, p. 43-59.

JAMES, C. (1987), « Some evidence on the uniqueness of bank loans », Journal of Financial Economics, vol. 19, p. 217-235.

JAMES, C. (1995), «When do banks take equity in debt restructurings?», The Review of Financial Studies, vol. 8, no 4, p. 1209-1234.

JOFFRE, P. et S. WICKAM (1997), «Les atouts des entreprises moyennes », Revue française de gestion, vol. 116, p. 64-70.

Jullien, H. et B. PARANQUe (1995), «Financement des entreprises et évolution du système financier », Revue internationale PME, vol. 8, $\mathrm{n}^{\text {os }} 3-4$, p. 43-66.

LACHMANN, J. (1996), Financer l'innovation des PME, Paris, Economica.

LefeBVRE, E., L. LEFEBVRE et M. BourgaulT (1996), « Performance à l'exportation et innovation technologique dans les PME manufacturières », Revue d'économie industrielle, vol. 77, p. 53-72.

LeVratto, N. (1994), «Le financement de l'innovation dans les PMI », Revue d'économie industrielle, vol. 67, p. 191-210.

LogossaH, K. (1994), «Capital humain et croissance économique : une revue de la littérature »,Économie et prévision, dans Problèmes économiques (1997), $\mathrm{n}^{\text {os }} 2510-2511$.

LUCAS, R.E. (1988), «On the mechanics of development planning », Journal of Monetary Economics, vol. 22, no 1, p. 3-42.

Muet, P.A. (1994), Croissance et cycles, Paris, Economica.

Myers, S. et N. MAJLuf (1984), «Corporate financing and investment decisions when firms have information that investors do not have », Journal of Financial Economics, vol. 13, p. 187-221.

PARANQUE, B. (1994), «Fonds propres, rentabilité et efficacité chez les PMI», Revue d'économie industrielle, vol. 67, p. 175-190. 
PARANQUe, B. et S. CiePly (1997), «Le rationnement des petites entreprises sur le marché du crédit : mythe ou réalité ?», Document de recherche présenté à l'AFFI, juin, p. $1-40$.

PICORY, C. (1995), «Organisation industrielle, degré d'intégration bancaire des PME et analyse du risque », Revue internationale PME, vol. 8, $\mathrm{n}^{\text {os }} 3-4$, p. 91-120.

PSILlAKI, M. (1995), « Rationnement du crédit et PME : une tentative de mise en relation », Revue internationale PME, vol. 8, $\mathrm{n}^{\text {os }} 3-4$, p. 67-90.

RAJAN, R. (1992), «Insiders and outsiders : the choice between informed and arm's-length debt », The Journal of Finance, vol. 47, no 4, p. 1367-1400.

RAPPORT GUILLAUME (1998), « Rapport de mission sur la technologie et l'innovation », Paris, Ministère de l'Économie, des Finances et de l'Industrie.

RAPPORT RISE CONSEIL (1997), «Rapport sur le marché du capital-investissement en France », Paris, Ministère de l'Économie, des Finances et de l'Industrie.

RAVID, S.A. et M. SPIEGEL (1997), «Optimal financial contracts for a start-up with unlimited operating discretion », Journal of Financial and Quantitative Analysis, vol. 32, no 3, p. 269-286.

ROMER, P.R. (1990), «Endogenous technological change », Journal of Political Economy, vol. $98, n^{\circ} 5$, p. 1022-1037.

SADIGH, E. (1995), «Croissance endogène et école néo-classique », Document de travail du LATEC n ${ }^{\circ}$ 9604, Université de Bourgogne.

ST-PIERRE, J. et N. AlLEPOT (1998), «Le crédit bancaire aux PME: les banques discriminent-elles selon la taille ou selon le risque des entreprises emprunteuses?», Document de recherche présenté au CIFPME, octobre.

SESSI (1998), L'industrie française, Secrétariat d'État à l'Industrie.

SHESHINSKY, E. (1967), « Optimal accumulation with learning by doing », dans Karl Shell (dir.), Essays on the Theory of Optimal Economic Growth, Cambridge, Mass., MIT Press, p. 31-52.

SolOw, T.R. (1956), «A contribution to the theory of economic growth », Quarterly Journal of Economy, vol. 70, $\mathrm{n}^{\circ}$ 1, p. 65-94.

Stiglitz, J.E. (1985), «Money, credit and banking lecture », Journal of Money, Credit and Banking, vol. 17, $\mathrm{n}^{\circ}$ 2, p. 133-152.

VON THADDEN, E. (1995), « Long-term contracts, short-term investment and monitoring », Review of Economic Studies, vol. 62, p. 557-575.

Vos, A. (1992), «Differences in risk measurement for small unlisted businesses », The Journal of Small Business Finance, vol. 1, no 3, p. 255-267.

WELCH, I. (1997), «Why is bank debt senior? A theory of asymmetry and claim priority based on influence costs », The Review of Financial Studies, vol. 10, n 4, p. 1203-1236.

WILLIAMSON, O. (1988), «Corporate finance and corporate governance », The Journal of Finance, vol. 43, no 3 , p. 567-591.

Winton, A. (1995), «Costly state verification and multiple investors: the role of seniority », The Review of Financial Studies, vol. 8, no 1, p. 91-123. 